\title{
Editorial
}

\section{Role of Medical Ethics, Epidemiological Studies and Team-based Learning Approach in Medical Education in Improving Clinical Practice and Patient Care}

Medical ethics is a systematic application of a set of moral principles that impart value to clinical practice and research. Once followed, these principles fulfill the expectations of the community and society (who is served and has diverse social and religious values) from the medical professionals within medical institutions. This approach consists of application of atleast seven basic principles: non-maleficence, beneficence, respect for person's autonomy, justice, proportionality, health maximization, efficiency, which are nonhierarchical (meaning no one principle routinely "trumps" another) and mutually exclusive, and may help one to fulfill their ethical obligations towards their patients or the subjects to be cared (1).

It appears that such principles in current usage in healthcare ethics are of self-evident value and can be clearly applied. It is generally held that these principles can be applied, even in unique circumstances to provide guidance in discovering our moral duties within those situations. For example, the principle that the physician "ought not to harm" any patient is on its face convincing to most people. The idea that the physician should develop a care plan designed to provide the most benefit to the patient in terms of other competing alternatives, seems both to be rational and self-evident. Further, before implementing the medical plan, it is now commonly accepted that the patient must be given an opportunity to make an informed choice about his or her care. Finally medical benefits should be dispensed fairly and proportionately so that people with similar needs and in similar circumstances will be treated with fairness, an important concept in the light of scarce resources such as solid organs and bone marrow transplants, expensive diagnostics, procedures and medications.

Intuitively, in the face of no other competing claims, we have a duty to uphold each of these principles (a prima facie duty). However, in the actual situation, we must balance the demands of these principles by determining which carries more weight in the particular case. Moral philosopher, WD Ross, claims that prima facie duties are always binding unless they are in conflict with other stronger or more stringent duties. A moral person's actual duty is determined by weighting and balancing all competing prima facie duties in any particular situation (2). Since principles are empty of contents, the application of the principle comes into focus through understanding the unique features and facts that provide the context for the case. Therefore, obtaining the relevant and accurate facts is an essential component of this approach to decision making (1).

The first paper in the present Issue of the Annals by Dr. Shridhar Sharma discusses some of the ethical dilemmas and their solutions when applied in dealing with the health of the subject in question, specially when this is an area of mental health (3). It has been argued that most ethical principles are still evolving and posing problems in every health care facility and are a challenge to every physician. 
In this context, the Mental Healthcare Act (MHCA), 2017 is a historical intervention which, if implemented well, can prove to be a game-changer. Two specific features stand out. First, the act adopts a rights-based approach. It places obligations on mental health services and prescribes procedures that ensure that mental health professionals offer treatment in accordance with a person's will and preferences. The law provides for the right to make advance directives- a person may state how they wish to be treated (or not) in the eventuality that they have a mental illness and cannot make decisions for themselves at that point of time. Additionally, the law recognizes an entire gamut of rights relating to confidentiality, access to medical records, protection from cruel treatment and nondiscrimination based on social markers, including sexual orientation. These rights are to be protected at all times when a person is undergoing mental healthcare and treatment as an admitted patient or otherwise. Two other Papers by Balhara et al (4) and Saimbi et al (5) are related to mental health issues of another kind, e.g. behavioural addiction and flower phobia and suggest the measures to treat them and how institutions like AIIMS, Delhi are making efforts to manage cases of behavioural addiction.

Following the multidisciplinary approach of the journal, the four other papers deal with three important aspects affecting the treating physicians (6-8) and medical educators (9). A number of research and review papers have shown the role of vitamin D (8) and gene therapy (6) in a variety of diseases including cardiovascular- myocardial infarction and stroke healing, diabetes mellitus-regeneration of beta-cells with pancreatic islets of Langerhans and psychosomatic disorders (7) as discussed in some of these papers are a few examples to cite. As the readers will find the claims for the success of these treatment modalities are many but the hard evidence in their favour is much meager. Regarding vitamin $\mathrm{D}$, it is a misnomer. It is not a true vitamin (an amine as the group name suggests), but a hormone, because it can be synthesized endogenously through ultraviolet exposure to skin. This sunlight triggered reaction produces cholecalciferol in skin; liver then convert it to calcidiol (25hydroxycholecalciferol/vitamin D2) which is then is converted by kidney to calcitriol $(1,25$ dihydroxycholecalciferol/vitamin D3) - the active physiological form of the D-hormone in the body. It is, thus a sacosteroid hormone that exists in three forms, that are sequential metabolites produced by hydroxylases.

As a fat soluble hormone, vitamin D hormone metabolites have a special mechanisms for delivery in aqueous blood stream- endogenously synthesized forms are carried by a binding protein, whereas dietary forms are carried within lipoprotein particles. These two different carrier pathways lead to distinct biodistribution for endogenously-derived versus supplement-derived vitamin D hormones and may be the reasons for discrepancies observed when compared the physiological effects and various organ system studied after giving vitamin D hormone/supplementations. Same logic can also be applied when discrepent results are obtained when one studies physiological role of genes in growth and development as opposite to when gene therapy approaches are used for healing or regeneration of cells after they are lost due to degenerative or injurious nature of the disease process.

\section{References}

1. McCormick TR (2013). Principles of bioethics. In: Ethics in Medicine. Washington: University ofWashington(bioethix@u.washingtom.edu). 
(iii)

2. Frankena WK (1973). Ethics, 2nd edn. Englewood Cliffs, NJ: Prentice-Hall.

3. Sharma S (2017). Evolving ethical issues in health and mental health. Ann Natl Acad Med Sci 53(3): 121-129.

4. Balhara YPS, Bhargava R, Chadda RK (2017). Service development for behavioural addictions: AIIMS experience. Ann Natl Acad Med Sci 53(3): 131-139.

5. Saimbi D, Sarmah SR, Kumar A, Shivalkar RP, Prasad S (2017). A curious case of flower phobia: anthophobia. Ann Natl Acad Med Sci 53(3): 175-178.

6. Thakur N, Batra P, Singh K, Gupta P (2017). Gene therapy in India-current status. Ann Natl Acad Med Sci 53(3): 140-147.

7. Sood M, Singh SK, Chadda RK (2017). Relevance of traditional Indian medical concepts in psychosomatic medicine. Ann Natl Acad Med Sci 53(3): 148-155.

8. Parveen R, Mishra P, Singh R, Kapur P, Agarwal NB (2017). Association of vitamin D and diabetic retinopathy in patients with type 2 diabetes mellitus. Ann Natl Acad Med Sci 53(3): 156-165.

9. Lal V, Bhandari B, Gupta G, Singh K, Sharma P (2017). Introducing the team based learning as an approach to reviving interest in biochemistry amongst undergraduate medical students- an exploratory study. Ann Natl Acad Med Sci 53(3): 166-174. 